\title{
An example of a non-Markovian stochastic two-point boundary value problem
}

\author{
MARCO FERRANTE ${ }^{1 *}$ and DAVID NUALART ${ }^{2}$ \\ ${ }^{1}$ Dipartimento di Matematica, Università di Padova, via Belzoni 7, 35100 Padova, Italy \\ ${ }^{2}$ Facultat de Matemàtiques, Universitat de Barcelona, Gran Via 585, 08007 Barcelona, Spain
}

In this paper we first present a multidimensional version of the characterization of the conditional independence in terms of a factorization property proved by Alabert et al. in the scalar case. As an application, we prove that the solution of a particular two-dimensional linear stochastic differential equation with boundary condition, considered by Ocone and Pardoux, is not a Markov field.

Keywords: boundary value problems; conditional independence; Markov field property; stochastic differential equations

\section{Introduction}

Consider the following $d$-dimensional stochastic differential equation of Stratonovich type:

$$
\mathrm{d} X_{t}=\left[A X_{t}+a(t)\right] \mathrm{d} t+\sum_{i=1}^{k}\left[B_{i} X_{t}+b_{i}(t)\right] \circ \mathrm{d} W_{t}^{i}, \quad 0 \leqslant t \leqslant 1,
$$

with the affine boundary condition

$$
F_{0} X_{0}+F_{1} X_{1}=f .
$$

Ocone and Pardoux (1989) provide necessary and sufficient conditions for the existence and uniqueness of a solution and establish sufficient conditions for the solution to be a Markov field. The Markov field property was studied by means of the co-area formula. In the Gaussian case (i.e., $B_{i}=0$ for all $i$ ) the Markov field property is always true. In the nonGaussian case a sufficient condition for the process $\left\{X_{t}\right\}$ to be a Markov field is that $a=b_{1}=\ldots=b_{k}=0$, and $\Phi_{t} \Phi_{s}^{-1}$ is a diagonal matrix for all $0 \leqslant s \leqslant t \leqslant 1$, where $\Phi_{t}$ is the $d \times d$ matrix-valued process solution of

$$
\mathrm{d} \Phi_{t}=A \Phi_{t} \mathrm{~d} t+\sum_{i=1}^{k} B_{i} \Phi_{t} \circ \mathrm{d} W_{t}^{i}, \quad 0 \leqslant t \leqslant 1, \quad \Phi_{0}=I d .
$$

\footnotetext{
*To whom correspondence should be addressed. e-mail: ferrante@math.unipd.it
} 
Nevertheless the Markov field property for a general process solution to (1.1) and (1.2) was left as an open problem by Ocone and Pardoux (1989).

In a recent paper (Alabert et al. 1995), we proved a characterization of the conditional independence of two independent random variables given a particular function of them, in terms of a factorization property. As an application of this result, we studied the Markov field property for solutions of stochastic differential equations with boundary conditions at the end-points of the time interval. The approach developed by Alabert et al. (1995) allowed us to extend some of the results obtained by Nualart and Pardoux (1991) and Donati-Martin (1991) using the method of change in probability.

Our aim here is to use the approach introduced by Alabert et al. (1995) to prove that there exists a stochastic differential equation with boundary conditions of the form (1.1) whose solution is not a Markov field. The counterexample will be the following twodimensional stochastic differential equation:

$$
\mathrm{d} X_{t}=\left(\begin{array}{ll}
1 & 1 \\
0 & 0
\end{array}\right) X_{t} \circ \mathrm{d} W_{t}^{1}+\left(\begin{array}{ll}
0 & 0 \\
0 & 1
\end{array}\right) X_{t} \circ \mathrm{d} W_{t}^{2}
$$

$t \in[0,1]$, with the boundary condition

$$
\left(\begin{array}{ll}
1 & 1 \\
0 & 0
\end{array}\right) X_{0}+\left(\begin{array}{ll}
0 & 0 \\
1 & 1
\end{array}\right) X_{1}=\left(\begin{array}{l}
1 \\
1
\end{array}\right) .
$$

This equation was considered by Ocone and Pardoux (1989), but with their technique, based on the co-area formula, they were not able to say whether or not the solution was a Markov field.

To prove that the solution to (1.3) and (1.4) is not a Markov field we shall make use of a characterization of the conditional independence of $\sigma$-fields in terms of a factorization property (Theorem 2.1). The particular example studied in this paper provides an illustration of the general method developed by Alabert et al. (1995) to check that the Markov property fails. The main result of the paper is proved in Section 3. In addition to Theorem 2.1 we shall apply the techniques of the anticipating stochastic calculus.

\section{Conditional independence of $\sigma$-fields and factorization properties}

In this section we shall give the definitions of local independence of $\sigma$-fields and local conditional independence of two $\sigma$-fields with respect to a third $\sigma$-field. Moreover we shall provide a multidimensional version of Theorem 2.1 of Alabert et al. (1995), which characterizes the conditional independence in terms of a factorization property.

Let $(\Omega, \mathscr{F}, P)$ be a probability space and for any sub- $\sigma$-field $\mathscr{G}$ of $\mathscr{F}$ and $B \in \mathscr{F}$ let us denote by $\mathscr{G}_{\mid B}$ the trace of $\mathscr{G}$ on $B$ defined by $\mathscr{G}_{\mid B}=\{A \cap B, A \in \mathscr{G}\}$. Note that, if $\mathscr{G}$ is generated by a random variable $X$, then $\mathscr{S}_{\mid B}=\sigma\left\{X_{\mid B}\right\}$, where $X_{\mid B}$ is the restriction of $X$ to the set $B$. We shall give the following definition of local independence of $\sigma$-fields. 
Definition 2.1. Let $\mathscr{F}_{1}$ and $\mathscr{F}_{2}$ be two sub- $\sigma$-fields of $\mathscr{F}$ and let $B \in \mathscr{F}$ with $P(B)>0$. We say that $\mathscr{F}_{1}$ and $\mathscr{F}_{2}$ are independent on the set $B$ if their traces $\mathscr{F}_{1 \mid B}$ and $\mathscr{F}_{2 \mid B}$ are independent on $\left(B, \mathscr{F}_{B}, P(\cdot \mid B)\right)$.

Clearly two $\sigma$-fields can be locally independent on some set $B$ without being independent. On the other hand, if two $\sigma$-fields are independent, then they may not be locally independent on some subset $B \in \mathscr{F}$, unless this set is of the form $B=B_{1} \cap B_{2}$, with $B_{1} \in \mathscr{F}_{1}$ and $B_{2} \in \mathscr{F}_{2}$.

It is interesting to point out that the local independence of two $\sigma$-fields $\mathscr{F}_{1}$ and $\mathscr{F}_{2}$ on a covering $\left\{B_{i}, i \in I\right\}$ of disjoint sets of $\Omega$, does not imply the independence of $\mathscr{F}_{1}$ and $\mathscr{F}_{2}$.

Let us now introduce the notion of local conditional independence. In the sequel we shall write

$$
\mathscr{F}_{1} \coprod_{\mathscr{G}} \mathscr{F}_{2}
$$

to mean that the $\sigma$-fields $\mathscr{F}_{1}$ and $\mathscr{F}_{2}$ are conditionally independent given the $\sigma$-field $\mathscr{G}$.

Definition 2.2. Let $\mathscr{F}_{1}, \mathscr{F}_{2}$ and $\mathscr{G}$ be sub- $\sigma$-fields of $\mathscr{F}$ and let $B \in \mathscr{F}$ with $P(B)>0$. We say that $\mathscr{F}_{1}$ and $\mathscr{T}_{2}$ are conditionally independent with respect to $\mathscr{G}$ on $B$ if

$$
\mathscr{F}_{1 \mid B} \coprod_{\mathscr{S} \mid B} \mathscr{F}_{2 \mid B}
$$

Recall the following result (Rozanov 1982, p. 57):

Lemma 2.1. Let $\mathscr{C}_{1} \coprod_{\mathscr{B}_{0}} \mathscr{C}_{2}$ and let us assume that

$$
\mathscr{B}_{1} \subset \mathscr{b}_{1} \vee \mathscr{B}_{0}, \quad \mathscr{B}_{2} \subset \mathscr{C}_{2} \vee \mathscr{B}_{0}, \quad \mathscr{B}_{0} \subset \mathscr{B}_{1} \vee \mathscr{B}_{2}
$$

Then,

$$
\mathscr{C}_{1} \coprod_{\mathscr{B}_{1} \vee \mathscr{B}_{2}} \mathscr{A}_{2} .
$$

Proposition 2.1. Let $(\Omega, \mathscr{F}, P)$ be a probability space, $\mathscr{F}_{1}, \mathscr{F}_{2}$ and $\mathscr{G}$ three sub- $\sigma$-fields of $\mathscr{F}, B_{1} \in \mathscr{F}_{1}$ and $B_{2} \in \mathscr{F}_{2}$ such that $P(B)>0$, where $B=B_{1} \cap B_{2}$. Then

$$
\mathscr{F}_{1} \coprod_{\mathscr{G}} \mathscr{F}_{2} \Rightarrow \mathscr{F}_{1 \mid B} \coprod_{\mathscr{G}_{\mid B}} \mathscr{F}_{2 \mid B}
$$

Proof. From Lemma 2.1 we get

$$
\mathscr{F}_{1} \coprod_{\mathscr{G}} \mathscr{F}_{2} \Rightarrow \mathscr{F}_{1} \underset{\mathscr{G} \vee \sigma\left\{B_{1}, B_{2}\right\}}{ } \mathscr{F}_{2} .
$$

Since the traces of $\mathscr{G}$ and $\mathscr{G} \vee \sigma\left\{B_{1}, B_{2}\right\}$ on $B$ coincide, it is sufficient to prove (2.1) when $B \in \mathscr{G}$. That is, given $A_{1} \in \mathscr{F}_{1}$ and $A_{2} \in \mathscr{F}_{2}$, we have to show that

$$
\overline{\mathrm{E}}\left[1_{A_{1} \cap A_{2} \cap B} \mid \mathscr{G}_{\mid B}\right]=\overline{\mathrm{E}}\left[1_{A_{1} \cap B} \mid \mathscr{G}_{\mid B}\right] \overline{\mathrm{E}}\left[1_{A_{2} \cap B} \mid \mathscr{G}_{\mid B}\right] \text {, }
$$


where $\overline{\mathrm{E}}$ denotes the mathematical expectation on the space $\left(B, \mathscr{F}_{\mid B}, \bar{P}\right)$ and $\bar{P}=P(\cdot \mid B)$. Note that, if $X$ is a bounded random variable and $B \in \mathscr{G}$, then

$$
\overline{\mathrm{E}}\left[X \mid \mathscr{G}_{\mid B}\right]=\mathrm{E}[X \mid \mathscr{G}] \quad \text { a.s. on } B .
$$

From (2.3), the conditional independence of $\mathscr{F}_{1}$ and $\mathscr{F}_{2}$ given $\mathscr{G}$, and the fact that $\mathrm{E}\left[1_{B} \mid \mathscr{G}\right]=1$ a.s. on $B$, we get

$$
\begin{aligned}
\overline{\mathrm{E}}\left[1_{A_{1} \cap A_{2} \cap B} \mid \mathscr{G}_{\mid B}\right] & =\mathrm{E}\left[1_{A_{1} \cap A_{2} \cap B} \mid \mathscr{G}\right] \\
& =\mathrm{E}\left[1_{A_{1} \cap B_{1}} \mid \mathscr{G}\right] \mathrm{E}\left[1_{A_{2} \cap B_{2}} \mid \mathscr{G}\right] \\
& =\mathrm{E}\left[1_{A_{1} \cap B_{1}} \mid \mathscr{G}\right] \mathrm{E}\left[1_{A_{2} \cap B_{2}} \mid \mathscr{S}\right] \mathrm{E}\left[1_{B_{1} \cap B_{2}} \mid \mathscr{G}\right] \\
& =\left(\mathrm{E}\left[1_{A_{1} \cap B_{1}} \mid \mathscr{G}\right] \mathrm{E}\left[1_{B_{2}} \mid \mathscr{G}\right]\right)\left(\mathrm{E}\left[1_{A_{2} \cap B_{2}} \mid \mathscr{G}\right] \mathrm{E}\left[1_{B_{1}} \mid \mathscr{G}\right]\right) \\
& =\mathrm{E}\left[1_{A_{1} \cap B} \mid \mathscr{G}\right] \mathrm{E}\left[1_{A_{2} \cap B} \mid \mathscr{G}\right] \\
& =\overline{\mathrm{E}}\left[1_{A_{1} \cap B}|\mathscr{G}| B\right] \overline{\mathrm{E}}\left[1_{A_{2} \cap B} \mid \mathscr{S}_{\mid B}\right]
\end{aligned}
$$

a.s. on $B$ and the result is proved.

In general we cannot deduce that two $\sigma$-fields are conditionally independent if they are conditionally independent of the sets of some partition of the space $\Omega$. For this reason the localization procedure will be useful just for proving negative results, i.e., that two $\sigma$-fields are not conditionally independent, being sufficient to prove this on a subset of the form $B_{1} \cap B_{2}$.

We shall now state a multidimensional version of Theorem 2.1 of Alabert et al. (1995) on the characterization of conditional independence in terms of a factorization property. The proof would be analogous to that of Theorem 2.1 of Alabert et al. (1995).

Let $(\Omega, \mathscr{F}, P)$ be a probability space and $\mathscr{F}_{1}$ and $\mathscr{F}_{2}$ two independent sub- $\sigma$-fields of $\mathscr{F}$. Consider two functions $g_{1}: \mathbb{R}^{d} \times \Omega \rightarrow \mathbb{R}^{d}$ and $g_{2}: \mathbb{R}^{d} \times \Omega \rightarrow \mathbb{R}^{d}$, set $B(\epsilon)=\left\{x \in \mathbb{R}^{d}\right.$, $|x|<\epsilon\}$, and denote by $\lambda$ the Lebesgue measure on $\mathbb{R}^{d}$. We shall assume that $g_{i}$ is $\mathscr{B}\left(\mathbb{R}^{d}\right) \otimes \mathscr{F}_{i}$ measurable, for $i=1,2$. Let us introduce the following conditions on the functions $g_{i}$.

(H1) For every $x \in \mathbb{R}^{d}$ and $y \in \mathbb{R}^{d}$ the random vectors $g_{1}(y, \cdot)$ and $g_{2}(x, \cdot)$ possess absolutely continuous distributions and the function

$$
\delta(x, y)=\sup _{0<\epsilon<\epsilon_{0}}\left(\frac{1}{[\lambda(B(\epsilon))]^{2}} P\left\{\left|x-g_{1}(y)\right|<\epsilon,\left|y-g_{2}(x)\right|<\epsilon\right\}\right)
$$

is locally integrable in $\mathbb{R}^{2 d}$, for some $\epsilon_{0}>0$.

(H2) For almost all $\omega \in \Omega$, and for any $|\xi|<\epsilon_{0}$, $|\eta|<\epsilon_{0}$ the system

$$
\begin{aligned}
& x-g_{1}(y, \omega)=\xi, \\
& y-g_{2}(x, \omega)=\eta,
\end{aligned}
$$

has a unique solution $(x, y) \in \mathbb{R}^{2 d}$.

(H3) For almost all $\omega \in \Omega$, the functions $y \rightarrow g_{1}(y, \omega)$ and $x \rightarrow g_{2}(x, \omega)$ are con- 
tinuously differentiable and there exists a non-negative random variable $H$ such that $\mathrm{E}(H)<\infty$ and

$$
\sup _{\left|y-g_{2}(x, \omega)\right|<\epsilon_{0}}\left\{\left|\operatorname{det}\left[I-\nabla g_{1}(y, \omega) \nabla g_{2}(x, \omega)\right]\right|^{-1}\right\} \leqslant H(\omega), \quad \text { a.s. }
$$

where $\nabla g_{i}$ denotes the Jacobian matrix of $g_{i}$.

Hypothesis (H2) implies the existence of two random vectors $X$ and $Y$ determined by the system

$$
\begin{aligned}
X(\omega) & =g_{1}(Y(\omega), \omega), \\
Y(\omega) & =g_{2}(X(\omega), \omega) .
\end{aligned}
$$

Theorem 2.1. Let $g_{1}$ and $g_{2}$ be two functions satisfying the preceding hypotheses (H1)(H3). Then the following statements are equivalent.

(i) $\mathscr{F}_{1}$ and $\mathscr{F}_{2}$ are conditionally independent given the random variables $X, Y$.

(ii) There exist two functions $F_{i}: \mathbb{R}^{2 d} \times \Omega \rightarrow \mathbb{R}, i=1,2$, which are $\mathscr{B}\left(\mathbb{R}^{2 d}\right) \otimes \mathscr{F}_{i}$ measurable for $i=1,2$, such that

$$
\left|\operatorname{det}\left[I-\nabla g_{1}(Y) \nabla g_{2}(X)\right]\right|=F_{1}(X, Y, \omega) F_{2}(X, Y, \omega), \quad \text { a.s. }
$$

Remark 2.1. Some of the conditions appearing in the above hypotheses can be weakened or modified and the conclusion of Theorem 2.1 will continue to hold.

(i) In hypothesis (H3) we can replace $H(\omega)$ by $H_{1}(\omega) H_{2}(\omega)$, with $H_{i}(\omega) \mathscr{F}_{i}$ measurable for $i=1,2$, and assume only $H_{1}(\omega) H_{2}(\omega)<\infty$ a.s.

(ii) In (H1) the local integrability of the function $\delta(x, y)$ holds if the densities $f_{1}(y, z)$ and $f_{2}(x, z)$ of $g_{1}(y)$ and $g_{2}(x)$ are locally bounded in $\mathbb{R}^{d} \times \mathbb{R}^{d}$. Furthermore, if we assume that there exist two open subsets of $\mathbb{R}^{d}, V_{1}$ and $V_{2}$, such that $P\left\{(X, Y) \in V_{1} \times V_{2}\right\}=1$, with $X, Y$ defined by (2.5), then we can assume that (H1) holds just for $(x, y) \in V_{1} \times V_{2}$.

We conclude this section with the following lemma, whose proof may be found in Alabert et al. (1995, p. 1269).

Lemma 2.2. Consider two independent $\sigma$-fields $\mathscr{F}_{1}, \mathscr{F}_{2}$ on a probability space $(\Omega, \mathscr{T}, P)$, and two random variables $G_{1}, G_{2}$ such that $G_{i}$ is $\mathscr{F}_{i}$ measurable for $i=1,2$. The following statements are equivalent.

(a) There exist two random variables $H_{1}$ and $H_{2}$ such that $H_{i}$ is $\mathscr{F}_{i}$ measurable, $i=1,2$, and

$$
1-G_{1} G_{2}=H_{1} H_{2} .
$$

(b) $G_{1}$ or $G_{2}$ is constant a.s. 


\section{Application to a linear stochastic differential equation with boundary condition}

Let $W=\left\{\left(W_{t}^{1}, W_{t}^{2}\right), t \in[0,1]\right\}$ be a standard two-dimensional Wiener process. Consider the following system of stochastic differential equations with a linear boundary condition at the end points of the time interval $[0,1]$ :

$$
\begin{gathered}
\mathrm{d} X_{t}=\left(\begin{array}{ll}
1 & 1 \\
0 & 0
\end{array}\right) X_{t} \circ \mathrm{d} W_{t}^{1}+\left(\begin{array}{ll}
0 & 0 \\
0 & 1
\end{array}\right) X_{t} \circ \mathrm{d} W_{t}^{2}, \quad t \in[0,1], \\
X_{0}^{1}+X_{0}^{2}=1, \quad X_{1}^{1}+X_{1}^{2}=1,
\end{gathered}
$$

where $X_{t}=\left(X_{t}^{1}, X_{t}^{2}\right)$. In this section we shall prove that the solution to this equation is not a Markov field using Theorem 2.1 and a suitable localization procedure.

Because of the boundary condition, we cannot expect the solution to be adapted to the Wiener filtration and the circle on the right-hand side of (3.1) denotes the extended anticipating Stratonovich integral (see Nualart 1995 for more details). Ocone and Pardoux (1989) proved that (3.1) admits a unique solution that can be computed explicitly. To simplify the computations, let us make the following linear change in variables:

$$
\begin{aligned}
& Y_{t}=X_{t}^{1}+X_{t}^{2}, \\
& Z_{t}=X_{t}^{2} .
\end{aligned}
$$

Then $\left(Y_{t}, Z_{t}\right)$ is the solution to the following stochastic boundary value problem:

$$
\begin{gathered}
\mathrm{d} Y_{t}=Y_{t} \circ \mathrm{d} W_{t}^{1}+Z_{t} \circ \mathrm{d} W_{t}^{2}, \\
\mathrm{~d} Z_{t}=Z_{t} \circ \mathrm{d} W_{t}^{2}, \quad t \in[0,1], \\
Y_{0}=Y_{1}=1,
\end{gathered}
$$

and (3.3) is equivalent to (3.1). An easy computation yields the solution to (3.3) is the twodimensional process

$$
\begin{aligned}
& Y_{t}=\mathrm{e}^{W_{t}^{1}}+Z_{0} \int_{0}^{t} \mathrm{e}^{W_{t}^{1}-W_{r}^{1}} \mathrm{e}^{W_{r}^{2}} \circ \mathrm{d} W_{r}^{2}, \\
& Z_{t}=Z_{0} \mathrm{e}^{W_{t}^{2}},
\end{aligned}
$$

$t \in[0,1]$, where $Z_{0}$ is the random variable:

$$
Z_{0}=\frac{1-\mathrm{e}^{W_{1}^{1}}}{\int_{0}^{1} \mathrm{e}^{W_{1}^{1}-W_{r}^{1}} \mathrm{e}^{W_{r}^{2}} \circ \mathrm{d} W_{r}^{2}}=\frac{\mathrm{e}^{-W_{1}^{1}}-1}{\int_{0}^{1} \mathrm{e}^{W_{r}^{1}+W_{r}^{2}} \circ \mathrm{d} W_{r}^{2}} .
$$

Let us recall the definition of a Markov field. 
Definition 3.1. A continuous process $\left\{X_{t}, t \in[0,1]\right\}$ is said to be a Markov field if, for any $0 \leqslant s<t \leqslant 1$, the $\sigma$-fields $\sigma\left\{X_{r}, r \in[s, t]\right\}$ and $\sigma\left\{X_{u}, u \in[0, s] \cap[t, 1]\right\}$ are conditionally independent given $\sigma\left\{X_{s}, X_{t}\right\}$.

The main result of the present section is the following.

Theorem 3.1. The two-dimensional process $\left\{\left(X_{t}^{1}, X_{t}^{2}\right), t \in[0,1]\right\}$, solution of (3.1), is not a Markov field.

Before proving this theorem let us show a technical lemma.

Lemma 3.1. For all $t \in(0,1]$ the random vector

$$
R_{t}=\left(\mathrm{e}^{-W_{t}^{1}}, \mathrm{e}^{W_{t}^{2}}, \int_{0}^{t} \mathrm{e}^{-W_{r}^{1}+W_{r}^{2}} \circ \mathrm{d} W_{r}^{2}\right)
$$

possesses an infinitely differentiable density, and the support of the law of $R_{t}$ is $[0, \infty)^{2} \times \mathbb{R}$. Moreover the density of $R_{t}$ belongs to the Schwartz space of rapidly decreasing functions.

Proof. The stochastic process $\left\{R_{t}\right\}$ solves the following stochastic differential equation in $\mathbb{R}^{3}$ :

$$
\begin{aligned}
& \mathrm{d} R_{t}^{1}=-R_{t}^{1} \circ \mathrm{d} W_{t}^{1}, \\
& \mathrm{~d} R_{t}^{2}=R_{t}^{2} \circ \mathrm{d} W_{t}^{2}, \\
& \mathrm{~d} R_{t}^{3}=R_{t}^{1} R_{t}^{2} \circ \mathrm{d} W_{t}^{2},
\end{aligned}
$$

with the initial condition $R_{0}=(1,1,0)$. Note that the vector fields

$$
A_{1}=\left(\begin{array}{l}
-x^{1} \\
0 \\
0
\end{array}\right), \quad A_{2}=\left(\begin{array}{l}
0 \\
x^{2} \\
x^{1} x^{2}
\end{array}\right),
$$

satisfy the hypoellipticity Hörmander condition at point $(1,1,0)$ (see, for example, Ikeda and Watanabe 1989). In fact, we have that the Lie bracket between $A_{1}$ and $A_{2}$ is

$$
\left[A_{1}, A_{2}\right]=\left(\begin{array}{l}
0 \\
0 \\
-x^{1} x^{2}
\end{array}\right),
$$

and we see that the vectors $A_{1}(1,1,0), A_{2}(1,1,0)$ and $\left[A_{1}, A_{2}\right](1,1,0)$ span $\mathbb{R}^{3}$. Thus Hörmander's theorem implies that for any $t \in(0,1]$ the vector $R_{t}$ has an infinitely differentiable density belonging to the Schwartz space (see, for example, Stroock 1983). The fact that the support of the law of $R_{t}$ is $[0, \infty)^{2} \times \mathbb{R}$ is an immediate consequence of the support theorem of Stroock and Varadhan for diffusion processes (see, for example, Ikeda and Watanabe 1989). 
Proof of Theorem 3.1. We shall divide the proof into several steps. The first step is a straightforward consequence of the change in variables (3.2).

Step 1. The process $\left\{\left(X_{t}^{1}, X_{t}^{2}\right), t \in[0,1]\right\}$ is a Markov field if and only if the process $\left\{\left(Y_{t}, Z_{t}\right), t \in[0,1]\right\}$, solution of (3.3), is a Markov field.

Let us define the two $\sigma$-fields

$$
\begin{aligned}
& \mathscr{F}_{s, t}^{\mathrm{i}}=\sigma\left\{W_{r}-W_{s}, r \in[s, t]\right\}, \\
& \mathscr{F}_{s, t}^{\mathrm{e}}=\sigma\left\{W_{u}, u \in[0, s]\right\} \vee \sigma\left\{W_{1}-W_{u}, u \in[t, 1]\right\},
\end{aligned}
$$

for $0 \leqslant s<t \leqslant 1$.

Step 2. The process $\left\{\left(Y_{t}, Z_{t}\right), t \in[0,1]\right\}$ is a Markov field if and only if, for any $0 \leqslant s<$ $t \leqslant 1$,

$$
\mathscr{F}_{s, t}^{\mathrm{i}} \coprod_{\sigma\left\{Y_{s}, Z_{s}, Y_{t}, Z_{t}\right\}} \mathscr{F}_{s, t}^{\mathrm{e}} .
$$

Proof of Step 2. By Definition 3.1 the process $\left\{\left(Y_{t}, Z_{t}\right), t \in[0,1]\right\}$ is a Markov field if and only if

$$
\sigma\left\{\left(Y_{r}, Z_{r}\right), r \in[s, t]\right\} \underset{\sigma\left\{Y_{s}, Z_{s}, Y_{t}, Z_{t}\right\}}{\coprod_{u}} \sigma\left\{\left(Y_{u}, Z_{u}\right), u \in[0, s] \cup[t, 1]\right\}
$$

for any $0 \leqslant s<t \leqslant 1$.

We have to prove that, for any $0 \leqslant s<t \leqslant 1$, (3.7) is equivalent to (3.6). From the inclusions

$$
\begin{gathered}
\sigma\left\{\left(Y_{r}, Z_{r}\right), r \in[s, t]\right\} \subset \mathscr{F}_{s, t}^{\mathrm{i}} \vee \sigma\left\{Y_{s}, Z_{s}, Y_{t}, Z_{t}\right\}, \\
\sigma\left\{\left(Y_{u}, Z_{u}\right), u \in[0, s] \cup[t, 1]\right\} \subset \mathscr{F}_{s, t}^{\mathrm{e}} \vee \sigma\left\{Y_{s}, Z_{s}, Y_{t}, Z_{t}\right\},
\end{gathered}
$$

we have that (3.6) implies (3.7).

The converse implication will be a consequence of the two following inclusions:

$$
\begin{gathered}
\mathscr{F}_{s, t}^{\mathrm{i}} \subset \sigma\left\{\left(Y_{r}, Z_{r}\right), r \in[s, t]\right\}, \\
\mathscr{F}_{s, t}^{\mathrm{e}} \subset \sigma\left\{\left(Y_{u}, Z_{u}\right), u \in[0, s] \cup[t, 1]\right\} .
\end{gathered}
$$

We shall prove that (3.8) is true and the proof of (3.9) would follow the same lines.

From (3.3) we obtain that for all $r \in[s, t]$

$$
Z_{r}=Z_{s} \mathrm{e}^{W_{r}^{2}-W_{s}^{2}},
$$

which implies, using that $Z_{0} \neq 0$ a.s. (as a consequence of Lemma 3.1), that

$$
\sigma\left\{W_{r}^{2}-W_{s}^{2}, r \in[s, t]\right\} \subset \sigma\left\{Z_{r}, r \in[s, t]\right\} .
$$

In order to show (3.8) it remains to prove that 


$$
\sigma\left\{W_{r}^{1}-W_{s}^{1}, r \in[s, t]\right\} \subset \sigma\left\{\left(Y_{r}, Z_{r}\right), r \in[s, t]\right\},
$$

and we can assume that $0<s<t<1$. This would follow from the heuristic equality

$$
W_{r}^{1}-W_{s}^{1}=\int_{s}^{r} Y_{u}^{-1} \circ \mathrm{d} Y_{u}-\int_{s}^{r} Z_{u} Y_{u}^{-1} \circ \mathrm{d} W_{u}^{2}
$$

but, in order to give a formal proof, some technical work involving anticipating stochastic integrals is needed.

Consider the following adapted stochastic differential equation, for $r \in[s, t]$ :

$$
\begin{gathered}
\mathrm{d} Y_{r}\left(x_{1}, x_{2}\right)=Y_{r}\left(x_{1}, x_{2}\right) \circ \mathrm{d} W_{r}^{1}+Z_{r}\left(x_{1}, x_{2}\right) \circ \mathrm{d} W_{r}^{2}, \\
\mathrm{~d} Z_{r}\left(x_{1}, x_{2}\right)=Z_{r}\left(x_{1}, x_{2}\right) \circ \mathrm{d} W_{r}^{2}, \\
Y_{s}=x_{1}, \quad Z_{s}=x_{2} .
\end{gathered}
$$

Passing from the Stratonovich to the Itô form, we obtain, for each $r \in[s, t]$,

$$
\begin{gathered}
\mathrm{d} Y_{r}\left(x_{1}, x_{2}\right)=Y_{r}\left(x_{1}, x_{2}\right) \mathrm{d} W_{r}^{1}+Z_{r}\left(x_{1}, x_{2}\right) \mathrm{d} W_{r}^{2}+\frac{1}{2}\left[Y_{r}\left(x_{1}, x_{2}\right)+Z_{r}\left(x_{1}, x_{2}\right)\right] \mathrm{d} r, \\
\mathrm{~d} Z_{r}\left(x_{1}, x_{2}\right)=Z_{r}\left(x_{1}, x_{2}\right) \mathrm{d} W_{r}^{2}+\frac{1}{2} Z_{r}\left(x_{1}, x_{2}\right) \mathrm{d} r \\
Y_{s}=x_{1}, \quad Z_{s}=x_{2} .
\end{gathered}
$$

Our aim is to show that a stochastic integral of the form

$$
\int_{s}^{r} \phi\left(Y_{u}\right) Y_{u} \mathrm{~d} W_{u}^{1}, \quad r \in[s, t],
$$

is $\sigma\left\{\left(Y_{r}, Z_{r}\right), r \in[s, t]\right\}$ measurable for any function $\phi \in C_{b}^{\infty}(\mathbb{R})$. Then we shall take a sequence of functions $\phi_{n} \in C_{b}^{\infty}(\mathbb{R})$ converging to $1 / x$ on $\{x \neq 0\}$ in order to deduce the measurability of the increments of $W^{1}$ on $[s, t]$ with respect to $\sigma\left\{\left(Y_{r}, Z_{r}\right), r \in[s, t]\right\}$. Given $\phi \in C_{b}^{\infty}(\mathbb{R})$ we define

$$
\psi(y)=\int_{0}^{y} \phi(x) \mathrm{d} x .
$$

Applying the Itô formula to $\psi\left(Y_{r}\right)$ we get

$$
\begin{aligned}
\mathrm{d} \psi\left(Y_{r}\left(x_{1}, x_{2}\right)\right)= & \phi\left(Y_{r}\left(x_{1}, x_{2}\right)\right) \mathrm{d} Y_{r}\left(x_{1}, x_{2}\right)+\frac{1}{2} \phi^{\prime}\left(Y_{r}\left(x_{1}, x_{2}\right)\right)\left[Y_{r}^{2}\left(x_{1}, x_{2}\right)+Z_{r}^{2}\left(x_{1}, x_{2}\right)\right] \mathrm{d} r \\
= & \phi\left(Y_{r}\left(x_{1}, x_{2}\right)\right) Y_{r}\left(x_{1}, x_{2}\right) \mathrm{d} W_{r}^{1}+\phi\left(Y_{r}\left(x_{1}, x_{2}\right)\right) Z_{r}\left(x_{1}, x_{2}\right) \mathrm{d} W_{r}^{2} \\
& +\frac{1}{2}\left[\phi\left(Y_{r}\left(x_{1}, x_{2}\right)\right)\left[Y_{r}\left(x_{1}, x_{2}\right)+Z_{r}\left(x_{1}, x_{2}\right)\right]\right. \\
& \left.\quad+\phi^{\prime}\left(Y_{r}\left(x_{1}, x_{2}\right)\right)\left[Y_{r}^{2}\left(x_{1}, x_{2}\right)+Z_{r}^{2}\left(x_{1}, x_{2}\right)\right]\right] \mathrm{d} r .
\end{aligned}
$$

Note that $Y_{r}\left(x_{1}, x_{2}\right)$ and $Z_{r}\left(x_{1}, x_{2}\right)$ are linear functions of $\left(x_{1}, x_{2}\right)$, and we have that $\left(Y_{r}\left(x_{1}, x_{2}\right), Z_{r}\left(x_{1}, x_{2}\right)\right)_{\mid\left(x_{1}, x_{2}\right)=\left(Y_{s}, Z_{s}\right)}=\left(Y_{r}, Z_{r}\right)$. For any $[a, b] \subset[s, t]$ the stochastic integrals

$$
\int_{a}^{b} \phi\left(Y_{r}\left(x_{1}, x_{2}\right)\right) Y_{r}\left(x_{1}, x_{2}\right) \mathrm{d} W_{r}^{1}
$$


and

$$
\int_{a}^{b} \phi\left(Y_{r}\left(x_{1}, x_{2}\right)\right) Z_{r}\left(x_{1}, x_{2}\right) \mathrm{d} W_{r}^{2}
$$

have continuous versions in the parameter $\left(x_{1}, x_{2}\right)$. This follows easily from Kolmogorov's continuity criterion. From (3.13) we get

$$
\begin{aligned}
& \int_{s}^{r} \phi\left(Y_{\theta}\left(x_{1}, x_{2}\right)\right) Y_{\theta}\left(x_{1}, x_{2}\right) \mathrm{d} W_{\theta \mid\left(x_{1}, x_{2}\right)=\left(Y_{s}, Z_{s}\right)}^{1} \\
&=\psi\left(Y_{r}\right)-\psi\left(Y_{s}\right)-I_{s}^{r}-\frac{1}{2} \int_{s}^{r}\left[\phi\left(Y_{\theta}\right)\left(Y_{\theta}+Z_{\theta}\right)+\phi^{\prime}\left(Y_{\theta}\right)\left(Y_{\theta}^{2}+Z_{\theta}^{2}\right)\right] \mathrm{d} \theta,
\end{aligned}
$$

where

$$
I_{s}^{r}=\int_{s}^{r} \phi\left(Y_{\theta}\left(x_{1}, x_{2}\right)\right) Z_{\theta}\left(x_{1}, x_{2}\right) \mathrm{d} W_{\theta \mid\left(x_{1}, x_{2}\right)=\left(Y_{s}, Z_{s}\right)}^{2} .
$$

Note that

$$
I_{s}^{r}=\lim _{|\boldsymbol{\pi}| \downarrow 0} \sum_{i=0}^{n-1} \phi\left(Y_{t_{i}}\right) Z_{t_{i}}\left(W_{t_{i+1}}^{2}-W_{t_{i}}^{2}\right),
$$

where $\pi=\left\{s=t_{0}<t_{1}<\cdots<t_{n}=t\right\}$, and the convergence holds in probability (Russo and Vallois 1993). Therefore we obtain that

$$
\int_{s}^{r} \phi\left(Y_{\theta}\left(x_{1}, x_{2}\right)\right) Y_{\theta}\left(x_{1}, x_{2}\right) \mathrm{d} W_{\theta \mid\left(x_{1}, x_{2}\right)=\left(Y_{s}, Z_{s}\right)}^{1}
$$

is measurable with respect to $\sigma\left\{\left(Y_{r}, Z_{r}\right), r \in[s, t]\right\}$.

We claim that $\left\{W_{r}^{1}-W_{s}^{1}, r \in[s, t]\right\}$ is a semimartingale with respect to the enlarged filtration $\left\{\mathscr{F}_{s, r}^{\mathrm{i}} \vee \sigma\left\{Y_{s}, Z_{s}\right\}, r \in[s, t]\right\}$ and, as a consequence, (3.14) coincides with the semimartingale integral

$$
\int_{s}^{r} \phi\left(Y_{u}\right) Y_{u} \mathrm{~d} W_{u}^{1}
$$

which implies that this integral is measurable with respect to $\sigma\left\{\left(Y_{r}, Z_{r}, r \in[s, t]\right\}\right.$. By Theorem 1.1 of Jacod (1985), the process $\left\{W_{r}^{1}-W_{s}^{1}, r \in[s, t]\right\}$ is a semimartingale with respect to the filtration $\left\{\mathscr{F}_{s, r}^{\mathrm{i}} \vee \sigma\left\{Y_{s}, Z_{s}\right\}, r \in[s, t]\right\}$ if for each $r \in[s, t]$ the law of $\left(Y_{s}, Z_{s}\right)$ conditioned by $\mathscr{F}_{s, r}^{\mathrm{i}}$ is absolutely continuous with respect to the Lebesgue measure on $\mathbb{R}^{2}$. Let us check this absolute continuity property. It suffices to show that the law of $\left(Y_{s}, Z_{s}\right)$ conditioned by $\mathscr{F}_{s, t}^{\mathrm{i}}$ is absolutely continuous with respect to the Lebesgue measure on $\mathbb{R}^{2}$. Taking into account (3.4) and (3.5), the vector $\left(Y_{s}, Z_{s}\right)$ can be written as $T(A, B)$, where $A$ and $B$ are the random vectors given by

$$
A=\left(\mathrm{e}^{-W_{s}^{1}}, \mathrm{e}^{W_{s}^{2}}, \int_{0}^{s} \mathrm{e}^{-W_{r}^{1}+W_{r}^{2}} \circ \mathrm{d} W_{r}^{2}, \mathrm{e}^{-\left(W_{1}^{1}-W_{t}^{1}\right)}, \int_{t}^{1} \mathrm{e}^{-\left(W_{r}^{1}-W_{t}^{1}\right)+\left(W_{r}^{2}-W_{t}^{2}\right)} \circ \mathrm{d} W_{r}^{2}\right),
$$




$$
B=\left(\mathrm{e}^{-\left(W_{t}^{1}-W_{s}^{1}\right)}, \mathrm{e}^{W_{t}^{2}-W_{s}^{2}}, \int_{s}^{t} \mathrm{e}^{-\left(W_{r}^{1}-W_{s}^{1}\right)+\left(W_{r}^{2}-W_{s}^{2}\right)} \circ \mathrm{d} W_{r}^{2}\right)
$$

and $T$ is the transformation

$$
T\left(x_{1}, x_{2}, x_{3}, x_{4}, x_{5}, \alpha_{1}, \alpha_{2}, \alpha_{3}\right)=\left(\frac{x_{2}\left(\alpha_{3}+x_{5} \alpha_{1} \alpha_{2}\right)+x_{3} x_{4} \alpha_{1}}{x_{3}+x_{1} x_{2}\left(\alpha_{3}+x_{5} \alpha_{1} \alpha_{2}\right)}, \frac{x_{1} x_{2} x_{4} \alpha_{1}-x_{2}}{x_{3}+x_{1} x_{2}\left(\alpha_{3}+x_{5} \alpha_{1} \alpha_{2}\right)}\right) .
$$

Note that $B$ is $\mathscr{F}_{s, t}^{\mathrm{i}}$ measurable and $A$ is independent of $\mathscr{F}_{s, t}^{\mathrm{i}}$. Then the absolute continuity of the law of $\left(Y_{s}, Z_{s}\right)$ conditioned by $\mathscr{F}_{s, t}^{\mathrm{i}}$ follows from the fact that $A$ has an infinitely differentiable density due to Lemma 3.1 and the transformation $T$ verifies $\operatorname{det}\left[\nabla_{x} T\left(\nabla_{x} T\right)^{t}\right]>0$ a.e., where $\nabla_{x} T$ denotes the Jacobian matrix of $T$ in the variables $\left(x_{1}, \ldots, x_{5}\right)$. In order to show the positivity of this determinant we fix $\left(\alpha_{1}, \alpha_{2}, \alpha_{3}\right) \in(0,+\infty)^{2} \times \mathbb{R}$ and consider the change in variables

$$
\begin{aligned}
& \bar{x}_{5}=\alpha_{3}+\alpha_{1} \alpha_{2} x_{5}, \\
& \bar{x}_{4}=\alpha_{1} x_{4}, \\
& \bar{x}_{3}=\left(x_{3}+x_{1} x_{2} \bar{x}_{5}\right)^{-1}, \\
& \bar{x}_{2}=x_{2} \bar{x}_{3}, \\
& \bar{x}_{1}=x_{1} \bar{x}_{2} .
\end{aligned}
$$

Then it suffices to observe that $\operatorname{det}\left(\nabla \bar{T}(\nabla \bar{T})^{t}\right)>0$ a.e., where

$$
\bar{T}\left(\bar{x}_{1}, \bar{x}_{2}, \bar{x}_{3}, \bar{x}_{4}, \bar{x}_{5}\right)=\left(\bar{x}_{2} \bar{x}_{5}+\bar{x}_{4}-\bar{x}_{1} \bar{x}_{4} \bar{x}_{5}, \bar{x}_{1} \bar{x}_{4}-\bar{x}_{2}\right) .
$$

Indeed, a simple computation yields

$$
\operatorname{det}\left[\nabla \bar{T}(\nabla \bar{T})^{t}\right]=\left(1+\bar{x}_{4}\right)^{2}+\left(\bar{x}_{4}^{2}+\bar{x}_{1}^{2}+1\right)\left(\bar{x}_{2}-\bar{x}_{1} \bar{x}_{4}\right)^{2} .
$$

So we have proved that $\int_{s}^{r} \phi\left(Y_{u}\right) Y_{u} \mathrm{~d} W_{u}^{1}$ is $\sigma\left\{\left(Y_{r}, Z_{r}\right), r \in[s, t]\right\}$ measurable for all $r \in[s, t]$ and $\phi \in C_{b}^{\infty}(\mathbb{R})$.

Consider now a sequence of smooth functions $\phi_{n} \in C_{b}^{\infty}(\mathbb{R})$ such that $\phi_{n}(x)=1 / x$ if $|x|>1 / n$, and $\left|\phi_{n}(x)\right| \leqslant n+1$ for all $x$. We have that

$$
\phi_{n}\left(Y_{u}\right) Y_{u} \rightarrow 1_{\left\{Y_{u} \neq 0\right\}} \quad \text { a.s. }
$$

By the dominated convergence theorem for stochastic integrals with respect to a semimartingale (Protter 1990), we obtain that

$$
\int_{s}^{r} \phi_{n}\left(Y_{u}\right) Y_{u} \mathrm{~d} W_{u}^{1}
$$

converges in probability to

$$
\int_{S}^{r} 1_{\left\{Y_{u} \neq 0\right\}} \mathrm{d} W_{u}^{1},
$$


which is therefore $\sigma\left\{\left(Y_{r}, Z_{r}\right), r \in[s, t]\right\}$ measurable. We have that $P\left\{Y_{u}=0\right\}=0$, for all $u \in(0,1]$. This implies that the last integral coincides with $W_{r}^{1}-W_{s}^{1}$ and (3.10) holds. Hence the proof of Step 2 is complete.

We are going to use the following notation. For any $0 \leqslant a<b \leqslant 1$ set

$$
L_{a, b}=\int_{a}^{b} \mathrm{e}^{-\left(W_{r}^{1}-W_{a}^{1}\right)+\left(W_{r}^{2}-W_{a}^{2}\right)} \circ \mathrm{d} W_{r}^{2} .
$$

Note that $L_{a, b}$ has the same distribution as $R_{b-a}^{3}$, where $R_{t}$ is the stochastic process introduced in Lemma 3.1. Define, for $0<s<t<1$,

$$
\begin{aligned}
& B_{1}=\left\{-2<W_{s}^{1}<-1,1<W_{s}^{2}<2,1<L_{0, s}<2\right\}, \\
& B_{2}=\left\{-2<W_{t}^{1}-W_{s}^{1}<-1,1<W_{t}^{2}-W_{s}^{2}<2,1<L_{s, t}<2\right\}, \\
& B_{3}=\left\{-2<W_{1}^{1}-W_{t}^{1}<-1,1<W_{1}^{2}-W_{t}^{2}<2,1<L_{t, 1}<2\right\} .
\end{aligned}
$$

Note that $B_{2} \in \mathscr{F}_{s, t}^{\mathrm{i}}, B_{1}, B_{3} \in \mathscr{F}_{s, t}^{\mathrm{e}}$, and $B_{1}$ and $B_{3}$ are independent. Moreover, as a consequence of Lemma 3.1 we have that $P\left(B_{i}\right)>0$ for $i=1,2$, 3. Set

$$
B=B_{1} \cap B_{2} \cap B_{3} \text {. }
$$

Step 3. Fix $0<s<t<1$. The conditional independence (3.6) implies the following factorization property. There exist two maps $F_{i}: \mathbb{R}^{4} \times B \rightarrow \mathbb{R}, i=1,2$, where $B$ is the set defined in (3.15), which are measurable with respect to the $\sigma$-fields $\mathscr{B}\left(\mathbb{R}^{4}\right) \otimes \mathscr{F}_{s, t \mid B}^{\mathrm{i}}$ and $\mathscr{B}\left(\mathbb{R}^{4}\right) \otimes \mathscr{F}_{s, t \mid B}^{\mathrm{e}}$, respectively, such that

$$
1+\frac{\mathrm{e}^{-W_{t}^{2}+W_{t}^{1}}}{L_{t, 1}}\left(\mathrm{e}^{W_{s}^{2}-W_{s}^{1}} L_{s, t}+L_{0, s}\right)=F_{1}\left(Y_{s}, Z_{s}, Y_{t}, Z_{t}\right) F_{2}\left(Y_{s}, Z_{s}, Y_{t}, Z_{t}\right), \quad \text { a.s. }
$$

Proof of Step 3. The result will be a consequence of Theorem 2.1 applied to the probability space $\left(B, \mathscr{F}_{\mid B}, P_{\mid B}\right)$. In order to apply this theorem we have to determine two maps:

$$
g_{1}, g_{2}: \mathbb{R}^{2} \times B \rightarrow \mathbb{R}^{2}
$$

satisfying the assumptions (H1), (H2) and (H3), such that $g_{1}$ is $\mathscr{B}\left(\mathbb{R}^{2}\right) \otimes \mathscr{F}_{s, t \mid B}^{\mathrm{i}}$ measurable, $g_{2}$ is $\mathscr{B}\left(\mathbb{R}^{2}\right) \otimes \mathscr{F}_{s, t \mid B}^{\mathrm{e}}$ measurable and, for almost every $\omega \in B$,

$$
\begin{aligned}
& \left(Z_{s}(\omega), Y_{t}(\omega)\right)=g_{1}\left(Y_{s}(\omega), Z_{t}(\omega), \omega\right), \\
& \left(Y_{s}(\omega), Z_{t}(\omega)\right)=g_{2}\left(Z_{s}(\omega), Y_{t}(\omega), \omega\right) .
\end{aligned}
$$

Remark 3.1. In comparison with other applications of the factorization method (Alabert et al. 1995, Section 4), in the present case we combine the components of the random vectors $\left(Y_{s}, Z_{s}\right)$ and $\left(Y_{t}, Z_{t}\right)$. In fact here we are able to determine $\left(Z_{s}, Y_{t}\right)$ as a function of $\left(Y_{s}, Z_{t}\right)$ and $\left\{W_{r}-W_{s}, r \in[s, t]\right\}$ and $\left(Y_{s}, Z_{t}\right)$ as a function of $\left(Z_{s}, Y_{t}\right)$ and $\left\{W_{u}, u \in[0, s]\right\}$, $\left\{W_{1}-W_{u}, u \in[t, 1]\right\}$. 
The system (3.17) holds with the following affine functions:

$$
\begin{aligned}
& g_{1}\left(y_{1}, y_{2}\right)=\left(y_{2} \mathrm{e}^{-\left(W_{t}^{2}-W_{s}^{2}\right)}, \mathrm{e}^{W_{t}^{1}-W_{s}^{1}}\left(y_{1}+y_{2} \mathrm{e}^{-\left(W_{t}^{2}-W_{s}^{2}\right)} L_{s, t}\right)\right), \\
& g_{2}\left(x_{1}, x_{2}\right)=\left(\mathrm{e}^{W_{s}^{1}}+x_{1} \mathrm{e}^{W_{s}^{1}-W_{s}^{2}} L_{0, s},\left(\mathrm{e}^{-\left(W_{1}^{1}-W_{t}^{1}\right)}-x_{2}\right)\left(L_{t, 1}\right)^{-1}\right) .
\end{aligned}
$$

We have to check that the maps $g_{1}$ and $g_{2}$ comply with the assumptions (H1)-(H3). The Jacobian matrices of mappings $g_{1}$ and $g_{2}$ are

$$
\nabla_{y} g_{1}=\left[\begin{array}{cc}
0 & \mathrm{e}^{-\left(W_{t}^{2}-W_{s}^{2}\right)} \\
\mathrm{e}^{W_{t}^{1}-W_{s}^{1}} & \mathrm{e}^{\left(W_{t}^{1}-W_{s}^{1}\right)-\left(W_{t}^{2}-W_{s}^{2}\right)} L_{s, t}
\end{array}\right]
$$

and

$$
\nabla_{x} g_{2}=\left[\begin{array}{cc}
\mathrm{e}^{W_{s}^{1}-W_{s}^{2}} L_{0, s} & 0 \\
0 & -\left(L_{t, 1}\right)^{-1}
\end{array}\right]
$$

A simple computation gives

$$
\operatorname{det}\left[I-\nabla_{y} g_{1} \nabla_{x} g_{2}\right]=1+\frac{\mathrm{e}^{-W_{t}^{2}+W_{t}^{1}}}{L_{t, 1}}\left(\mathrm{e}^{W_{s}^{2}-W_{s}^{1}} L_{s, t}+L_{0, s}\right) .
$$

On the set $B$ this determinant is larger than 1 and hypothesis (H3) holds.

To prove (H2), it will be sufficient to show that for all $\omega \in B$ the linear map on $\mathbb{R}^{n}$

$$
\Gamma_{\omega}\left(x_{1}, x_{2}, y_{1}, y_{2}\right)=\left(\left(x_{1}, x_{2}\right)-g_{1}\left(y_{1}, y_{2}, \omega\right),\left(y_{1}, y_{2}\right)-g_{2}\left(x_{1}, x_{2}, \omega\right)\right)
$$

is bijective. This is a consequence of the fact that for all $\omega \in B$ we have

$$
\operatorname{det}\left[\nabla_{x, y} \Gamma_{\omega}\right]=\operatorname{det}\left[I-\nabla_{y} g_{1}\left(y_{1}, y_{2}, \omega\right) \nabla_{x} g_{2}\left(x_{1}, x_{2}, \omega\right)\right]>1 .
$$

Finally, in order to show condition (H1) we have to see that the laws of the random variables $g_{1}\left(y_{1}, y_{2}\right)$ and $g_{2}\left(x_{1}, x_{2}\right)$ conditioned by the set $B$ possess densities which are locally bounded in all their variables. Note that there exist constants $0<a_{1}<a_{2}<0$ such that on the set $B$ we have $a_{1} \leqslant Y_{s}, Z_{s}, Y_{t}, Z_{t} \leqslant a_{2}$. Let us denote the densities of $g_{1}\left(y_{1}, y_{2}\right)$ and $g_{2}\left(x_{1}, x_{2}\right)$ by $f_{g_{1}\left(y_{1}, y_{2}\right)}\left(z_{1}, z_{2}\right)$ and $f_{g_{2}\left(x_{1}, x_{2}\right)}\left(z_{1}, z_{2}\right)$, respectively. By Remark 2.1 it is sufficient to show that the functions $f_{g_{1}\left(y_{1}, y_{2}\right)}\left(z_{1}, z_{2}\right)$ and $f_{g_{2}\left(x_{1}, x_{2}\right)}\left(z_{1}, z_{2}\right)$ are bounded when $\left(y_{1}, y_{2}, z_{1}, z_{2}\right)$ or $\left(x_{1}, x_{2}, z_{1}, z_{2}\right)$ belong to $\left[a_{1}^{\prime}, a_{2}^{\prime}\right]$ where $0<a_{1}^{\prime}<a_{2}^{\prime}$. Note first that $g_{1}\left(y_{1}, y_{2}\right)$ has the same law as

$$
\left(y_{2}\left(R_{t-s}^{2}\right)^{-1}, y_{1}\left(R_{t-s}^{1}\right)^{-1}+y_{2}\left(R_{t-s}^{1}\right)^{-1}\left(R_{t-s}^{2}\right)^{-1} R_{t-s}^{3}\right),
$$

where $\left\{R_{t}, t \in[0,1]\right\}$ is the stochastic process introduced in Lemma 3.1. Denote the density of $R_{t}$ by $\rho_{t}\left(r_{1}, r_{2}, r_{3}\right)$. Then the density of $g_{1}\left(y_{1}, y_{2}\right)$ will be

$$
\begin{aligned}
f_{g_{1}\left(y_{1}, y_{2}\right)}\left(z_{1}, z_{2}\right) & =\int_{0}^{\infty} \rho_{t-s}\left(\frac{y_{1}}{\theta}, \frac{y_{2}}{z_{1}}, \frac{y_{1}\left(z_{2}-\theta\right)}{\theta z_{1}}\right) \frac{y_{1}^{2} y_{2}}{z_{1}^{3} \theta^{3}} \mathrm{~d} \theta \\
& =\int_{0}^{\infty} \rho_{t-s}\left(y_{1} \xi, \frac{y_{2}}{z_{1}}, \frac{y_{1}}{z_{1}}\left(z_{2} \xi-1\right)\right) \frac{y_{1}^{2} y_{2}}{z_{1}^{3}} \xi \mathrm{d} \xi,
\end{aligned}
$$


and this is bounded when $\left(y_{1}, y_{2}, z_{1}, z_{2}\right) \in\left[a_{1}^{\prime}, a_{2}^{\prime}\right]$ because by Lemma 3.1 we have $\rho_{t-s}\left(r_{1}, r_{2}, r_{3}\right) \leqslant c_{n} /\left(r_{1}^{2}+r_{2}^{2}+r_{3}^{2}\right)^{n}$ for each $n \geqslant 1$, where $c_{n}$ is a constant.

The random vector $g_{2}\left(x_{1}, x_{2}\right)$ would be treated in a similar way.

To complete the proof it will be sufficient to prove that (3.16) leads to a contradiction.

Step 4. As in Step 3 fix $0<s<t<1$. Then, the factorization property (3.16) does not hold.

Proof of Step 4. Let us rewrite (3.16) in terms of $\left(Y_{s}, Z_{s}, Y_{t}, Z_{t}\right)$. From (3.4) we have

$$
\begin{aligned}
Z_{0} \mathrm{e}^{W_{1}^{1}-W_{t}^{1}+W_{t}^{2}} L_{t, 1} & =1-Y_{t} \mathrm{e}^{W_{1}^{1}-W_{t}^{1}}, \\
Z_{0} \mathrm{e}^{W_{t}^{1}-W_{s}^{1}+W_{s}^{2}} L_{s, t} & =Y_{t}-Y_{s} \mathrm{e}^{W_{t}^{1}-W_{s}^{1}}, \\
Z_{0} \mathrm{e}^{W_{s}^{1}} L_{0, s} & =Y_{s}-\mathrm{e}^{W_{s}^{1}} .
\end{aligned}
$$

Substituting these equalities in (3.16) yields

$$
1+\frac{\mathrm{e}^{W_{1}^{1}-W_{t}^{1}}}{1-Y_{t} \mathrm{e}^{W_{1}^{1}-W_{t}^{1}}}\left(Y_{t}-\mathrm{e}^{W_{t}^{1}}\right)=F_{1}\left(Y_{s}, Z_{s}, Y_{t}, Z_{t}\right) F_{2}\left(Y_{s}, Z_{s}, Y_{t}, Z_{t}\right), \quad \text { a.s. on } B \text {. }
$$

A simple computation gives

$$
1-\mathrm{e}^{W_{1}^{1}}=F_{1} F_{2}\left(1-Y_{t} \mathrm{e}^{W_{1}^{1}-W_{t}^{1}}\right), \quad \text { a.s. on } B,
$$

where the map $F_{2}\left(x_{1}, x_{2}, x_{3}, x_{4}\right)\left(1-x_{3} \mathrm{e}^{W_{1}^{1}-W_{t}^{1}}\right)$ is still $\mathscr{B}\left(\mathbb{R}^{4}\right) \otimes \mathscr{F}_{s, t \mid B}^{\mathrm{e}}$ measurable.

If we set $G_{1}=\mathrm{e}^{W_{1}^{1}-W_{t}^{1}} \mathrm{e}^{W_{s}^{1}}$ and $G_{2}=\mathrm{e}^{W_{t}^{1}-W_{s}^{1}}$, it follows from Lemma 2.2 that (3.19) implies the following property: one (or both) of the random variables $G_{1}$ and $G_{2}$ is almost surely constant on $B$ with respect to the conditional law given $\left(Y_{s}, Z_{s}, Y_{t}, Z_{t}\right)$. This condition holds only if there exists a measurable function $h: \mathbb{R}^{4} \rightarrow \mathbb{R}$ such that

$$
\mathrm{e}^{W_{1}^{1}-W_{t}^{1}} \mathrm{e}^{W_{s}^{1}}=h\left(Y_{s}, Z_{s}, Y_{t}, Z_{t}\right) \quad \text { a.s. on } B
$$

or

$$
\mathrm{e}^{W_{t}^{1}-W_{s}^{1}}=h\left(Y_{s}, Z_{s}, Y_{t}, Z_{t}\right) \quad \text { a.s. on } B .
$$

To find a contradiction it suffices to show that the random vectors

$$
A_{1}=\left(Y_{s}, Z_{s}, Y_{t}, Z_{t}, \mathrm{e}^{W_{s}^{1}}, \mathrm{e}^{W_{1}^{1}-W_{t}^{1}}\right)
$$

and

$$
A_{2}=\left(Y_{s}, Z_{s}, Y_{t}, Z_{t}, \mathrm{e}^{W_{t}^{1}-W_{s}^{1}}\right)
$$

possess absolutely continuous distributions. This follows easily from the fact that these vectors can be obtained as transformations of the vector

$$
C=\left(\mathrm{e}^{-W_{s}^{1}}, \mathrm{e}^{W_{s}^{2}}, L_{0, s}, \mathrm{e}^{-\left(W_{t}^{1}-W_{s}^{1}\right)}, \mathrm{e}^{W_{t}^{2}-W_{s}^{2}}, L_{s, t}, \mathrm{e}^{-\left(W_{1}^{1}-W_{t}^{1}\right)}, \mathrm{e}^{W_{1}^{2}-W_{t}^{2}}, L_{t, 1}\right)
$$

Note that the components of $C$ are independent and with the same distributions as $R_{s}^{1}, R_{s}^{2}$, 
$R_{s}^{3}, R_{t-s}^{1}, R_{t-s}^{2}, R_{t-s}^{3}, R_{1-t}^{1}, R_{1-t}^{2}, R_{1-t}^{3}$ where $\left\{R_{t}\right\}$ is the stochastic process introduced in Lemma 3.1. We have $A_{1}=T_{1}(C)$ and $A_{2}=T_{2}(C)$, where

$$
\begin{aligned}
& T_{1}(x)=\left(\frac{1}{x_{1}}+\frac{x_{3}}{x_{1}} \Phi(x), x_{2} \Phi(x), \frac{1+\left(x_{3}+x_{1} x_{2} x_{6}\right) \Phi(x)}{x_{1} x_{4}}, x_{2} x_{5} \Phi(x), \frac{1}{x_{1}}, \frac{1}{x_{7}}\right), \\
& T_{2}(x)=\left(\frac{1}{x_{1}}+\frac{x_{3}}{x_{1}} \Phi(x), x_{2} \Phi(x), \frac{1+\left(x_{3}+x_{1} x_{2} x_{6}\right) \Phi(x)}{x_{1} x_{4}}, x_{2} x_{5} \Phi(x), \frac{1}{x_{4}}\right)
\end{aligned}
$$

and

$$
\Phi(x)=\frac{x_{1} x_{4} x_{7}-1}{x_{3}+x_{1} x_{2} x_{6}+x_{1} x_{2} x_{4} x_{5} x_{9}} .
$$

Then the absolute continuity of the laws of $A_{1}$ and $A_{2}$ follows from that of the random vector $C$ (as a consequence of Lemma 3.1), and the property $\operatorname{det}\left[\nabla T_{i}\left(\nabla T_{i}\right)^{t}\right]>0$, a.e. for $i=1,2$. The positivity of these determinants can be proved by means of suitable change of variables. For instance, in the case of $T_{2}$, put

$$
\begin{aligned}
& \bar{x}_{7}=\Phi(x), \\
& \bar{x}_{2}=x_{2} \bar{x}_{7}, \\
& \bar{x}_{5}=x_{2} x_{5} \bar{x}_{7}, \\
& \bar{x}_{4}=\frac{1}{x_{4}}, \\
& \bar{x}_{3}=x_{1} x_{2} x_{6}+x_{3}, \\
& \bar{x}_{1}=\frac{1}{x_{1}}, \\
& \bar{x}_{6}=x_{6} .
\end{aligned}
$$

Then the problem reduces to check that $\operatorname{det}\left[\nabla \bar{T}_{2}\left(\nabla \bar{T}_{2}\right)^{t}\right]>0$, a.e. where

$$
\bar{T}_{2}(\bar{x})=\left(\bar{x}_{1}\left(1+\bar{x}_{7} \bar{x}_{3}\right)-\bar{x}_{2} \bar{x}_{6}, \bar{x}_{2},\left(1+\bar{x}_{3} \bar{x}_{7}\right) \bar{x}_{1} \bar{x}_{4}, \bar{x}_{5}, \bar{x}_{4}\right),
$$

and this is easy. The transformation $T_{1}$ is handled in the same way. The proof of Theorem 3.1 is now complete.

\section{Acknowledgements}

This paper was written while the first author was visiting the Universitat de Barcelona with a HCM fellowship of the European Union.

\section{References}

Alabert, A., Ferrante, M. and Nualart, D. (1995) Markov field property of stochastic differential equations. Ann. Probab., 23, 1262-1288. 
Donati-Martin, C. (1991) Équations différentielles stochastiques dans $\mathbb{R}$ avec conditions auxs bords. Stochastics Stoch. Reports, 35, 143-173.

Ikeda, N. and Watanabe, S. (1989) Stochastic Differential Equations and Diffusion Processes, 2nd edn. Amsterdam: North-Holland.

Jacod, J. (1985) Grossissement initial, hypothèse $(\mathrm{H})^{\prime}$, et théorème de Girsanov. In T. Jeulin and M. Yor (eds), Groississement de Filtrations: Exemples et Applications, pp. 15-35. Lecture Notes in Math. 1118. Berlin: Springer-Verlag.

Nualart, D. (1995) The Malliavin Calculus and Related Topics, Probability and its Applications. Berlin: Springer-Verlag.

Nualart, D. and Pardoux, E. (1991) Boundary value problems for stochastic differential equations, Ann. Probab., 19, 1118-1144.

Ocone, D. and Pardoux, E. (1989) Linear stochastic differential equations with boundary conditions. Probab. Theory Rel. Fields, 82, 439-526.

Protter, Ph. (1990) Stochastic Integration and Differential Equations. Appl. of Math. 21. Berlin: Springer-Verlag.

Rozanov, Yu. A. (1982) Markov Random Fields. Berlin: Springer-Verlag.

Russo, F. and Vallois, P. (1993) Forward, backward and symmetric stochastic integration, Probab. Theory Rel. Fields, 97, 403-421.

Stroock, D.W. (1983) Some applications of stochastic calculus to partial differential equations. In P.L. Hennequin (ed.), École d'Été de Probabilités de Saint-Flour, XI, pp. 267-382. Lecture Notes in Math. 976. Berlin: Springer-Verlag.

Received November 1995 and revised November 1996 